\title{
Searching Extra Dimensions in Compact Stars
}

\author{
G. G. Barnaföldi ${ }^{\star}$ P. Lévai, and B. Lukács \\ Research Institute for Particle and Nuclear Physics H-1525 Budapest, P.O.Box 49, Hungary
}

Received 20 March 2007, accepted xx March 2007

Published online later

Key words Cygnus $\mathrm{X} 3$, strange stars, compact star, neutron star, exotic hadrons, cygnets, $\mathrm{H}^{0}$ dibaryon, compactified extra dimensions, general relativity, Kaluza - Klein model

\begin{abstract}
The electro-magnetic and particle radiation detected from the direction of Cygnus X3 raise the question of the existence of special long-lived, neutral particles. We investigate the origin of these particles in a special approach: the source object may contain compactified extra dimension and these particles are messengers of this state. We describe hyperon stars in $3+1$ dimension and introduce the description of a compact stars in $3+1_{c}+1$ dimensional space-time.
\end{abstract}

(c) 2006 WILEY-VCH Verlag GmbH \& Co. KGaA, Weinheim

\section{Introduction}

The understanding of the Cygnus X3 is still in a shroud of mystery (Collins 2000). Although, both electro-magnetic radiations (radio, IR, optical, X-ray) and additional particle radiations (muons and neutrinos), have been observed since 1981 from the direction of the Cygnus X3 (J2000 $\alpha=308.1^{\circ}, \delta=40.96^{\circ}$ ), however the theoretical description of this object and the global understanding of the experimental data remained an open question even these days.

It is commonly accepted, that Cygnus X3 is a galactic, tight binary star system (with $\sim 5$ solar radii), which consists of a compact object $\left(\sim 3 M_{\odot}\right)$ and a $\sim 15 M_{\odot}$ mass companion Wolf-Rayet (WN7 or 8) star with huge mass loss. On the other hand, the Cygnus X3 is known as a 'micro-quasar', a small quasar in our galaxy, emitting strong radio flares and highly collimated relativistic jets towards Earth (Allison et al. 1998; Marshak et al. 1985, 2000).

Muon shower data have been collected between 1981 and 2002 by the deep underground muon detectors (e.g. SOUDAN, NUSEX, and SOUDAN2). The measured muon flux clearly correlates to the 4.8 hours X-ray cycle caused by the eclipsing (or wind) of the compact star by its companion (Hillas 1984). Furthermore, eruptions (flares) have been found episodically and their lengths are varying in a wide range, as collected in Table 1 .

In recent article we concentrate on the primary origin of the muon showers, if these showers are caused by particles produced in the Cygnus X3 and survived the trip to Earth. These particle-like objects are named 'cygnets', and they must have the following properties:

(i) neutral, otherwise the path would be curved by the galactic magnetic field which would have deflected their arrival directions or have randomized it;

\footnotetext{
* Corresponding author: e-mail: bgergely@ rmki.kfki.hu
}

Table 1 The main observed radio flares of Cygnus X3, based on the works of Allison et al. (1998), Marshak et al. (1985, 2000), Martí et al. (2001), and Miller-Jones et al. (2004).

\begin{tabular}{llc}
\hline Start (date) & End (date) & Duration (day) \\
\hline 1989 Jun 1 & 1989 Aug 14 & 75 \\
1990 Aug 11 & 1990 Aug 31 & 21 \\
1990 Oct 1 & 1990 Oct 22 & 22 \\
1991 Jan 18 & 1991 Mar 19 & 163 \\
1991 Jun 19 & 1991 Jun 30 & 12 \\
1991 Jul 24 & 1991 Aug 29 & 37 \\
1992 Sep 1 & 1992 Sep 11 & 11 \\
1994 Feb 20 & 1994 Mar 11 & 20 \\
1997 Feb 1 & 1997 Feb 16 & 16 \\
1997 Jun 10 & 1997 Jun 25 & 16 \\
1998 Mar 14 & 1998 Mar 20 & 7 \\
2000 Oct 21 & 2000 Oct 21 & 1 \\
2000 Nov 4 & 2000 Nov 5 & 2 \\
2000 Nov 19 & 2000 Nov 20 & 2 \\
2001 Sep 9 & 2001 Sep 26 & 18 \\
\hline
\end{tabular}

(ii) stable or long-lived, otherwise they would decay along their $10 \mathrm{kpc}(\sim 30,000$ ly) long way;

(iii) strongly-interacting, since they produce hadron showers including pions, which decay into muons.

New particles were suggested to solve the 'Cygnus X3puzzle': the 'uds-nuggets' proposed by Witten (1984); the ' $H^{0}$ dibaryons' listed by Baym (1985), Kondratiuk (1986), and BNL (2000). However these particles have not been detected yet experimentally.

In this paper we present another approach and study the appearence of microscopical quanta with extra dimensional aspect: these particles are produced inside the compact star, where usual description in $3+1$ dimensional space-time is extended by an extra Kaluza - Klein (KK) like compactified fifth dimension $\left(1_{c}\right)$. 


\section{Strangeness or Extra Dimension?}

The 'strange-star' aspect of the Cygnus X3 has been introduced and discussed several times (Grassi 1988; Olinto 1991; Glendenning 1997; Weber 1999): the basic idea is to assume a neutron or quark star with an inner core, which contains large number of strange quarks. Although, these strange quark stars usually become instable in the theoretical calculations from the point of view of radial excitations (Glendenning 1997; Prisznyák et al. 1994; Barnaföldi et al. 2003; Lukács et al. 2003), however quickly rotated quark stars may be stabilized. The existence of millisecond pulsars supports this expectation.

If such a strange quark star has a tight binary companion, which can reach the Roche lobe, then matter can flow to the compact object. The particles can gain enough kinetic energy and become relativistic, thus they can kick out matter below the neutron surface of the strange star. The out-kicked relativistic strange matter can fly away and reach our detectors in Earth. We claim that this mechanism is the most probable to create accelerated particle packages with extra strangeness content, which packages reach the atmosphere of the Earth time-to-time, and which packages are responsible for the measured intense muon showers. Since the muon showers are correlated with the X-ray outbursts of the Cygnus X3, then it is logical to assume that hadronic particle packages are created in the same mechanism as outbursts or flares. Thus we can introduce the name of 'hadronic $\mathrm{P}$ Cygni' for stars with similar behavior and which are detected in the proper optical category.

So far our study has been based on regular physics of hadrons and quarks. However, inside millisecond pulsars such extreme physical conditions may exist, when strong, electro-weak and gravitational interactions interplay with each other. It is challenging to describe the properties of these pulsars with unified models containing all of the general interactions. In this paper we introduce microscopical extra dimensions (Randall \& Sudrum 1999) and use the frame of a Kaluza-Klein like model, where the excited states of elementary particles appear as geometrical degrees of freedom (Kan \& Shiraishi 2000; Lukács et al. 2003). Space like extra dimension offers a unique possibility to introduce strangeness as an excitation generated by the light quark moving into such a compactified extra dimension. We will investigate, if the properties of quark stars are modified in the presence of compactified extra space dimensions.

\section{Motion into the $5^{\text {th }}$ Dimension}

Todays a new trend appeared in high energy physics, and the role of microscopical extra dimensions has been investigated from the point of view of high energy particle production. Although the symmetries of the observed 'low-energy' physical world suggest a space-time with $3+1$ macroscopical dimensions, we can not exclude the existence of compactified extra dimensions at microscopical scales. We can consider a $3+1_{c}+1$ dimensional space-time, where the particles have enough energy to move into the extra compactified space dimension indicated by $1_{c}$ (Barnaföldi et al. 2003, 2004; Lukács et al. 2003). Figure 1illustrates the appearance of a compactified dimension.

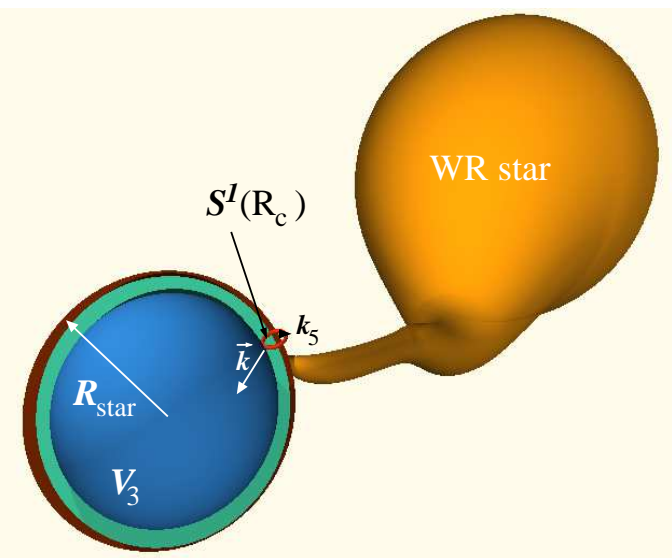

Fig. 1 Schematic view of the opening of the $5^{\text {th }}$ dimension on the Cygnus X3 binary system in a $3+1_{c}+1$ dimensional space-time, where the space has a $V_{3} \times S^{1}$ structure with a compactified radius $R_{c}$ of the $S^{1}$ circle. The momentum for moving in the $5^{\text {th }}$ dimension is denoted by $k_{5}$.

Particles can move freely along the extra $x^{5}$ direction, however we require a periodic boundary condition, which results a Bohr-type quantization condition for $k_{5}$ momentum component. This condition induces an uncertainty in the position with the size of $2 \pi R_{c}$, where $R_{c}$ is the compactification radius. Motion into the $5^{\text {th }}$ dimension generates an extra mass term appears in pure 4D description:

$$
k_{5}=\frac{n \hbar}{R_{c}} \quad \longrightarrow \quad \widehat{m}=\sqrt{m^{2}+\left(\frac{n \hbar}{R_{c}}\right)^{2}} .
$$

Considering compactified radius $R_{c} \sim 10^{-12}-10^{-13} \mathrm{~cm}$ this extra 'mass' is $\widehat{m} \sim 100 \mathrm{MeV}$, which is a familiar quantity in hadron spectroscopy (Arkhipov 2004).

One can introduce a 'pseudo charge' generated by $k_{5}$ with its \pm sign:

$$
\widehat{q}=n \cdot \frac{2 \hbar \sqrt{G}}{c R_{c}},
$$

which acts in a vector-scalar interaction. We can directly see that $\widehat{q}$ is not the electric charge. Indeed $\widehat{q}^{2}<16 \pi G m_{0}^{2}$, where $G$ is the gravitational constant and $m_{0}$ is the rest mass (Lukács \& Pacher 1985). This is either a familiar quantum number (e.g. strangeness, $S$ ), or another charge-like quanta, which is not yet observed experimentally.

\section{Field Equations in 5D}

Our goal is to describe the inner structure of a spherically symmetric quark star-like object in $3+1_{c}+1 \mathrm{D}$ space- 
time. Our object is static, stays in equilibrium, and its temperature is close to zero. We are using a perfect fluid description with isotropy in the standard 3 spatial dimensions. Killing-symmetries are still exist in 5D space-time, but an anisotropy needs to be chosen for the fluid in the microscopical $5^{\text {th }}$ dimension. These symmetries yield to the metric 1

$$
\mathrm{d} s^{2}=e^{2 \nu} \mathrm{d} t^{2}-e^{2 \lambda} \mathrm{d} r^{2}-r^{2} \mathrm{~d} \Omega^{2}-e^{2 \Phi}\left(\mathrm{d} x^{5}\right)^{2},
$$

where the quantities $\nu, \lambda$, and $\Phi$ depend only on the radius $r$. We denote the usual spherical elementary surface by $\mathrm{d} \Omega^{2}$. The 5D Einstein equation is written in the following way:

$$
-\gamma T_{i k}=R_{i k}-\frac{1}{2} R_{l}^{l} g_{i k} .
$$

Here the energy-momentum tensor contains the energy density $\varepsilon$, and the pressure components $P$ and $P_{5}$ (but $P \neq P_{5}$ ):

$$
T^{i k}=\operatorname{diag}\left(\varepsilon e^{2 \nu}, P e^{2 \lambda}, P r^{2}, P r^{2} \sin ^{2} \theta, P_{5} e^{2 \Phi}\right)
$$

Substituting eqs. (3) and (5) into the 5D Einstein equation in eq. (4) one obtains the following equation system:

$$
\begin{aligned}
& -\gamma \varepsilon=-\frac{1}{r^{2}}+ \\
& +e^{-2 \lambda}\left[\Phi^{\prime \prime}+\Phi^{\prime 2}-\lambda^{\prime} \Phi^{\prime}+\frac{2 \Phi^{\prime}}{r}-\frac{2 \lambda^{\prime}}{r}+\frac{1}{r^{2}}\right](6) \\
& -\gamma P=\frac{1}{r^{2}}+e^{-2 \lambda}\left[-\nu^{\prime} \Phi^{\prime}-\frac{2 \Phi^{\prime}}{r}-\frac{2 \nu^{\prime}}{r}-\frac{1}{r^{2}}\right](7) \\
& -\gamma P=e^{-2 \lambda}\left[-\nu^{\prime \prime}-\nu^{\prime 2}+\nu^{\prime} \lambda^{\prime}-\Phi^{\prime \prime}-\Phi^{\prime 2}-\right. \\
& \left.-\nu^{\prime} \Phi^{\prime}+\lambda^{\prime} \Phi^{\prime}-\frac{2 \Phi^{\prime}}{r}-\frac{\nu^{\prime}}{r}+\frac{2 \lambda^{\prime}}{r}\right] \\
& -\gamma P_{5}=\frac{1}{r^{2}}+ \\
& +e^{-2 \lambda}\left[-\nu^{\prime \prime}-\nu^{\prime 2}+\nu^{\prime} \lambda^{\prime}-\frac{2 \nu^{\prime}}{r}+\frac{2 \lambda^{\prime}}{r}-\frac{1}{r^{2}}\right](9)
\end{aligned}
$$

where $\gamma=8 \pi G / c^{4}$ and $G$ is the gravitational constant.

Assuming cold nuclear matter inside the fermion star ( $T=0$ approximation), all local material characteristics of the fluid depend on one thermodynamic quantity, e.g. particle density, $\rho$. Thus, for the local matter we can write

$$
\varepsilon=\varepsilon(\rho) ; \quad P=P(\rho) ; \quad P_{5}=P_{5}(\rho) .
$$

From the appropriate Bianchi identity we obtain

$$
T_{; r}^{i r}=0 \quad \longrightarrow \quad P^{\prime}=-\nu^{\prime}(\varepsilon+P)+\left(P_{5}-P\right) \Phi^{\prime} .
$$

This equation clearly demonstrates the influence of the extra dimensional behavior on the normal isotropic pressure, where $P=P_{1}=P_{2}=P_{3}$.

In the 5D Einstein equations (6) two extra variables appeared, compared to the 4D formalism, namely $P_{5}$ and $\Phi$. However, $P_{5}(\rho)$ is a known function of density and specified by the actual interaction in the matter. Thus $\Phi(r)$ is the only new degree of freedom determined by eq. (9). Further details can be found in (Barnaföldi, 2003).

1 Notations: we are using coordinates $x^{i}$ with Latin indices $(i=$ $0,1,2,3$ and 5 ) for $3+1_{c}+1 \mathrm{D}$ space-time with the Einstein convention.

\section{A Special 5D Solution}

We can recognize the similarity between 4D and 5D solutions. For specially chosen pressure component $P_{5}$, there is a unique solution of the Einstein equations in eqs. (6) 9], namely $\mathrm{d} \Phi / \mathrm{d} r=0$. In this case eqs. (6) 8) lead to the Tolman-Oppenheimer - Volkov equation (Glendenning 1997) and can be solved separately with the $\Phi=$ const. condition. The eq. (9) gives $P_{5}$. Although these solutions do not differ formally from the 4D neutron star solution (except for $P_{5}$ ), but the extra dimension has its influence on $\varepsilon(\rho)$ and $P(\rho)$ (Kan \& Shiraishi 2000). In case of $\Phi \neq$ const. condition, one must turn to numerics and solve directly eqs. (6. 9).

Neglecting the effects of electromagnetic charge, let us start with a neutral, single massive fermion ('neutron', $N$ ) as elementary building block of a compact star. Since the minimal nonzero fifth momentum component is given by Bohr-type quantization (1), then the extra direction of the phase space is not populated until the Fermi-momentum $k_{F}<\hbar / R_{c}$. However, at the threshold both $k_{5}= \pm \hbar / R_{c}$ states appear. One can represent this as another ('excited') particle with mass $m_{X}=\sqrt{m_{N}^{2}+\left(\hbar / R_{c}\right)^{2}}$ (with a nonelectric 'charge' $\widehat{q}= \pm \frac{2 \hbar \sqrt{G}}{c R_{c}}$ as well). The equations obtain a form as if this second particle appears in complete chemical equilibrium with the neutron: $\mu_{X}=\mu_{N}$.

This recipe is repeated for every integer $n$, when $k$ exceeds a threshold $n \hbar / R_{c}$, and these higher excitations are introduced into the equation of state.

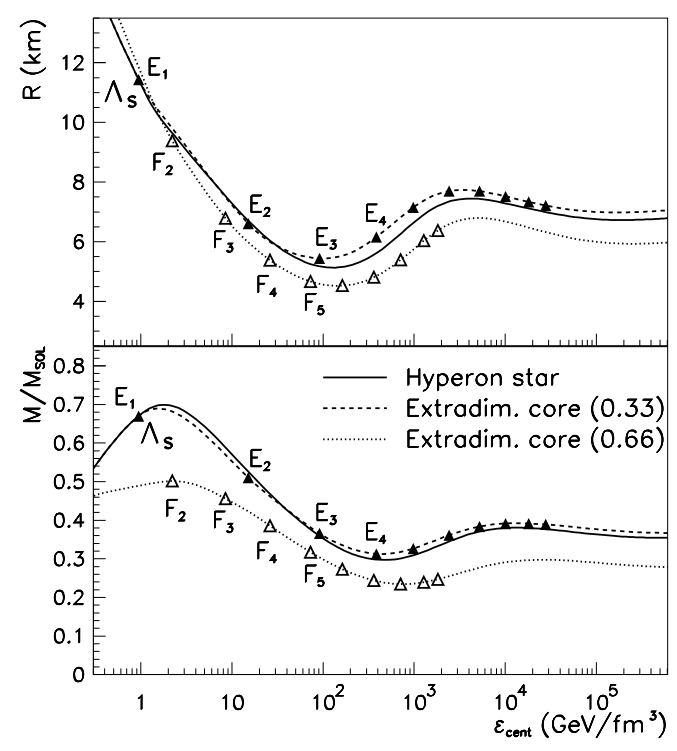

Fig. $2 M\left(\varepsilon_{\text {cent }}\right)$ and $R\left(\varepsilon_{\text {cent }}\right)$ functions for 4D hyperon star (solid lines) and 5D hybrid stars (dashed and dotted lines).

We apply our model for hybrid star with strangeness content as a reference. This object is a hyperon star in 4D with $N$ and $\Lambda_{s}$ content. After solving eqs. (6)-(8), Fig. 2 
displays the central energy density $\left(\varepsilon_{\text {cent }}\right)$ dependence of the star mass and star radius in this scenario (solid lines).

Next we introduce an extra compactified space dimension $\left(1_{c}\right)$ with radius $R_{c}=0.33 \mathrm{fm}$. In this case the particle $\Lambda_{s}$ can be interpreted as the first extra dimensional excited state of $N$. Solving eqs. (6)-(9) we obtain similar results to our reference hyperon star (dashed lines). The location of the appearances of extra dimensional excitations are denoted by the letters $E_{1}\left(\equiv \Lambda_{s}\right), E_{2}, E_{3}, \ldots$ on Fig. 2

Finally we vary the compactification radius and increase it to $R_{c}=0.66 \mathrm{fm}$. Solving the TOV-like equation system, we obtain slightly different curves for the star mass and radius (dotted lines), however the main characteristics remain the same. In Fig. 2 these slightly lighter extra-dimensional excitations are denoted by the letters $F_{1}, F_{2}, F_{3}, \ldots$.

We construct the $M(R)$ diagrams for all three scenarios and display them on Fig. 33 The introduction of a proper extra dimensional radius $\left(R_{c}=0.33 \mathrm{fm}\right)$ yields to a similar object to our reference hyperon star. This figure shows, that the change in the compactification radius is able to modify the $M(R)$ diagram. The study of the limits and consequences of these modifications are under investigation.

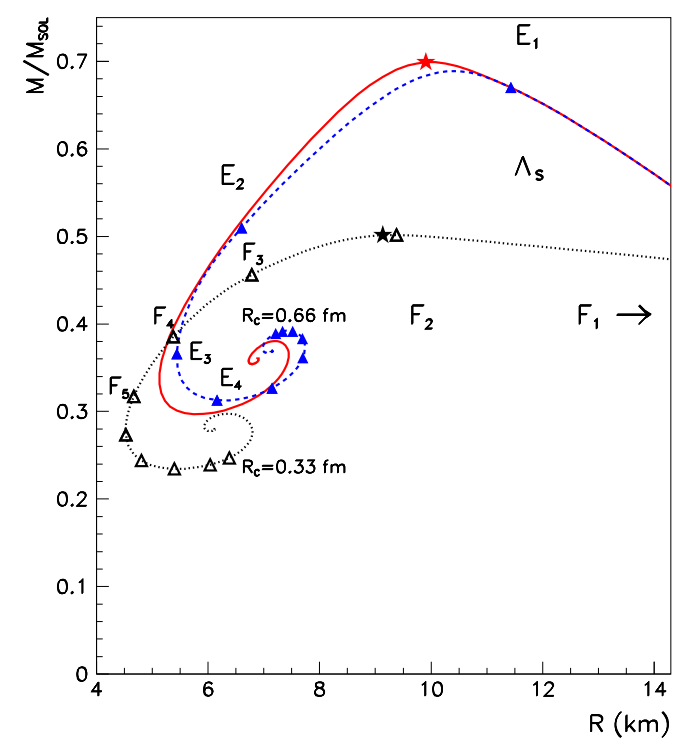

Fig. 3 The $M(R)$ diagrams for 4D hyperon star and 5D hybrid stars. See text for details.

\section{Discussion}

Introducing extra dimensions into the description of hybrid stars led to similar results in $3+1$ and $3+1_{c}+1$ spacetime. The $M(R)$ diagram does not change drastically, if the extra dimensional radius is in the same order as the strange quark mass difference $\left(\Delta m_{s} \sim \hbar c / R_{c}\right)$. However, the presence of one (or more) compactified dimension(s) may able to change the characteristics of these astrophys- ical objects. We are aware of the complexity of this question, and do not want to claim the extra dimensional origin of the strangeness. However such an astrophysical object as a compact or hybrid star serves as 'natural laboratory' to investigate these problems (Weber 1999) or other exotic ones, e.g. the flavor dependence of gravity (Fischbach et al. 1986, 1991). In the Earth the planned experiments at the Large Hadronic Collider (LHC) may shed of some light on this question in the near future. We are aware of the consideration of the rotation for the investigated astrophysical objects, but the correct calculation and the necessary statibility investigations require a very complex treatment, which is beyond the scope of our recent paper.

Acknowledgements. This work was supported by the Eötvös University Department of Astronomy, British Council and Hungarian OTKA grants T047050 and NK62044.

\section{References}

Allison et al.: 1999, Proc. of the $26^{\text {th }}$ Int. Cosmic Ray Conference (ICRC 99), Salt Lake City, Utah (arXiv:hep-ex/9905045)

Ambartsumyan, V. A., Saakyan, G. S.: 1960, Astron. Zh. 37, 193

Arkhipov, A. A.: 2004, AIP Conf. Proc. 717, 680

Aronson, S.H. et al.: 1986, Phys. Rev. Lett. 56, 1342

Barnaföldi, G. G., Lévai, P., Lukács, B.: 2003, in Proceedings of the 4th Int. Workshop on New Worlds in Astroparticle Physics, Faro, Portugal, Worlds Scientific, Singapoure;

Barnaföldi, G. G., Lévai, P., Lukács, B.: 2004, Publ. of the Astronomy Department of Astronomy of the Eötvös University, PADEU 14, Budapest, Hungary

Baym, G. et al.: 1985, Phys. Lett. 160B, 181

BNL - Brookhaven National Laboratory: 2000, See WEB-page http://www.phy.bnl.gov/newphysics/experiments.html

Collins A.: 2000, See WEB-page http://www.andrewcollins.com

Fischbach, E. et al.: 1986, Phys. Rev. Lett. 56, 3

Fischbach, E. et al.: 1996, Les Arcs 1996, Dark matter in cosmology, quantum measurements, experimental gravitation, 443

Glendenning, N. K.: 1997, Compact Stars, Springer, NH

Grassi, F.: 1988, FERMILAB-Pub-88/19-A

Hillas, A. M.: 1984, Ann. Rev. Astrophys, 22, 425

Kan, N., Shiraishi, K.: 2002, Phys. Rev. D66, 105014

Kondratiuk, L. A., Krivoruchenko, M. I., Shchepkin, M. G.: 1986, JETP Letters (ISSN 0021-3640), 43, 10

Lukács, B., Barnaföldi, G. G., Lévai, P.: 2003 in Proc. of NATO ARW on Superdense QCD Matter and Compact Stars, Yerevan, Armenia, NATO ASI Series 197, Blascke, D., Sedrakian, D. (eds.), Kluwer

Lukács, B.: 2000, Relativity Today, C. Hoenselaers, Z. Perjés (eds.), Akadémiai Kiadó, Budapest, 161

Lukács, B., Pacher, T.: 1985, Phys. Lett. A113, 200

Marshak, M. L. et al: 1985, Phys. Rev. Lett. 54, 2079 ibid 55, 1965

Marshak, M. L. et al.: 2000, Proc. of the International Conference of High Energy Physics (ICHEP 2000), see http://ichep2000.hep.sci.osaka-u.ac.jp/abs_PA-11.html

Martí, J., Paredes, J. M., Peracaula, M.: 2001, Astron. \& Astrophys. 375,476

Miller-Jones et al.: 2004, Astrophys. J. 600, 368

Olinto, A. V.: 1991, FERMILAB-Conf-91/202-A; 349-A

Prisznyák, M. Lukács B., Lévai, P.: 1994, Report KFKI-199424/A, KFKI, Budapest

Randall, L., Sudrum, R.: 1999, Phys. Rev. Lett. 83, 3370 ibid 4690 
Weber, F.: 1999, Astrophysical Laboratories for Nuclear and Particle Physics, IOP Publishing, Bristol, England

Witten, E.: 1984, Phys. Rev. D30, 272 